\title{
Endosymbiotic bacteria in insects: guardians of the immune system?
}

\section{Ioannis Eleftherianos*, Jaishri Atri, Julia Accetta and Julio C. Castillo}

Insect Infection and Immunity Lab, Department of Biological Sciences, Columbian College of Arts and Sciences, Institute for Biomedical Sciences, The George Washington University, Washington, DC, USA

\section{Edited by:}

Sassan Asgari, The University of

Queensland, Australia

Reviewed by:

Steven Sinkins, University of

Oxford, UK

Kostas Bourtzis, International

Atomic Energy Agency, Austria

Jeremy Brownlie, Griffith University,

Australia

*Correspondence:

loannis Eleftherianos, Insect Infection and Immunity Lab,

Department of Biological Sciences,

Columbian College of Arts and

Sciences, Institute for Biomedical

Sciences, The George Washington

University, 2023 G Street NW,

Washington, DC 20052, USA.

e-mail: ioannise@gwu.edu
Insects have evolved obligate, mutualistic interactions with bacteria without further transmission to other eukaryotic organisms. Such long-term obligate partnerships between insects and bacteria have a profound effect on various physiological functions of the host. Here we provide an overview of the effects of endosymbiotic bacteria on the insect immune system as well as on the immune response of insects to pathogenic infections. Potential mechanisms through which endosymbionts can affect the ability of their host to resist an infection are discussed in the light of recent findings. We finally point out unresolved questions for future research and speculate how the current knowledge can be employed to design and implement measures for the effective control of agricultural insect pests and vectors of diseases.

Keywords: insect, infection, immunity, endosymbiont, Wolbachia, Spiroplasma

\section{INTRODUCTION}

Insects comprise about $95 \%$ of all known animal species and are considered one of the most successful groups of living organisms on earth. They possess an extremely efficient immune system that allows them to deal with pathogenic infections. The insect immune system consists of a wide variety of defense mechanisms that act individually or in combination to prevent foreign organisms from entering the insect body or to suppress the growth and replication of pathogens once they gain access to host tissues.

The first line of defense is represented by the insect's epithelia, which serves as a barrier against biotic and abiotic factors, and produce local antimicrobial peptides (AMP) upon infection or wounding (Davis and Engström, 2012). The second line of defense is represented by the innate immune system that responds through a series of mechanisms. These include the systemic production of AMP mainly from the fat body (the insect equivalent to the mammalian liver) as the result of the transcriptional regulation of signaling pathways that are activated upon immune challenge (Ganesan et al., 2011); cellular responses by insect hemocytes (equivalent to mammalian white blood cells) that take part in immune surveillance, phagocytosis, and encapsulation of foreign intruders (Marmaras and Lampropoulou, 2009); melanization and coagulation or clotting of the hemolymph (the insect analog of vertebrate blood), which require the active form of the enzyme phenoloxidase and the participation of humoral and cellular factors that lead to the rapid production and deposition of melanin around wounds, and foreign invaders (Eleftherianos and Revenis, 2011); generation of high levels of reactive oxygen species (ROS) and AMP in epithelial cells as well as nitric oxide (NO) that is also involved in the regulation of innate immune responses to bacteria and parasites (Ryu et al., 2010; Royet, 2011), which in some cases is stimulated by the gut microbiota; RNA interference (RNAi) and inducible innate immune responses against viral pathogens (Kemp and Imler, 2009). In addition, physiological and ecological factors that include dietary nutrition and energy metabolism, feeding behavior, circadian rhythms, aging, mating success and reproductive activity, lifestyle, and immune priming also affect the host immune response to pathogenic challenges (Sadd and Schmid-Hempel, 2009; Schneider, 2009; Chambers and Schneider, 2012).

Apart from the native microbiota, insects also carry symbiotic bacteria that occupy specific cells and tissues within the host. These symbiotic microbes live under the pressure of an active immune system and therefore they must devise strategies that allow them to withstand the adverse effects of host immune defense mechanisms (Gross et al., 2009; Douglas, 2011; Weiss and Aksoy, 2011). Furthermore, exposure of insects to microbes at all stages of their life cycle could have shaped the insect immune system to fight infection against pathogenic organisms (Mateos et al., 2006). Recent findings strongly suggest that the presence of symbiotic bacteria in various insect species is associated with increased host resistance to various pathogens and parasites. The results of these studies are of particular interest for developing alternative strategies to transgenic approaches for the efficient management of noxious insects. 


\section{ENDOSYMBIOTIC BACTERIA IN INSECTS}

Almost all insects are associated with heritable endosymbiotic bacteria. Many endosymbionts are able to form a mutualistic relationship with their host and others can cause severe effects on various biological functions of their insect partner (Feldhaar and Gross, 2009). Primary endosymbionts are vertically transmitted from mother to offspring and they provide their hosts with specific nutritional compounds that are important for their survival and development. For example, Buchnera aphidicola endosymbiotic bacteria of the pea aphid, Acyrthosiphon pisum, synthesize essential amino acids that the aphids cannot receive from the plant sap. Similarly, Wigglesworthia glossinidia endosymbionts of tsetse flies (Glossina morsitans) produce essential vitamins that are not present in the vertebrate blood meal (Aksoy and Rio, 2005; Oliver et al., 2010). Insects have co-evolved with their primary endosymbionts for several million years; therefore their relationship is obligate. This means that insects lacking their bacteria are unable to grow and reproduce while the symbiotic bacteria are not viable in the absence of their host (Kikuchi, 2009). Primary endosymbionts are mainly found in bacteriocytes, which are specialized cells that provide nutrients to the bacteria and they are contained within the bacteriome (Bright and Bulgheresi, 2010). Secondary endosymbionts can be transmitted horizontally, vertically or via the environment. These are commensal bacteria that have evolved symbiotic relationships with their hosts more recently and can be found in the hemocoel (insect body cavity containing the hemolymph and organs) (Wernegreen, 2012). For example, Sodalis glossinidius bacteria are secondary symbionts in the tsetse fly, which are maternally transmitted to the progeny and can be found inter- and intra-cellularly in various tissues of the fly (Balmand et al., 2012). Sodalis was the first insect endosymbiont that was reported to be successfully isolated and cultured in vitro (Matthew et al., 2005).

The most commonly found facultative endosymbiotic bacteria are Wolbachia and Spiroplasma. Wolbachia is a group of maternally transmitted intracellular alpha-proteobacteria that infect a wide range of insects as well as filarial nematodes. These symbionts are able to manipulate the reproductive properties of their insect hosts by inducing parthenogenesis, male-killing, feminization and, most commonly, cytoplasmic incompatibility (Werren et al., 2008; Saridaki and Bourtzis, 2010). Spiroplasma symbionts are wall-less, motile, helical, gram-positive bacteria that associate both endocellularly and extracellularly with a variety of arthropods, particularly insects. Some species of Spiroplasma bacteria cause female-biased sex ratios of their host insects including Drosophila flies, ladybird beetles, and butterflies, as a result of selective death of the male offspring during embryogenesis (Regassa and Gasparich, 2006; Haselkorn, 2010). The consequence of the diversity of hosts and symbioses is that Wolbachia and Spiroplasma endosymbionts must evade a broad range of host immune defense mechanisms to ensure their survival and transmission, and the host must regulate the bacterial population to avoid the effects of pathogenicity or fitness costs. On the contrary, the absence of other heritable endosymbionts apart from Wolbachia and Spiroplasma in Drosophila perhaps highlights the ability of the fly immune system to control infections by other symbiotic bacteria (Mateos et al., 2006).

\section{INTERACTION OF ENDOSYMBIONTS WITH THE INSECT IMMUNE SYSTEM}

Wolbachia and Spiroplasma endosymbionts can be found in the insect hemolymph and thus they can interact directly with secreted molecules of the humoral immune response (Dobson et al., 1999; Haselkorn, 2010). A previous study examined the transcription of AMP genes in Drosophila simulans flies and Aedes albopictus mosquitoes carrying or lacking Wolbachia endosymbionts (Bourtzis et al., 2000). Results showed that Diptericin and Cecropin genes were not up-regulated in Wolbachia infected flies compared to uninfected controls. Similarly, Wolbachia infection failed to induce Defensin gene transcription in mosquitoes. These findings indicate that Wolbachia endosymbionts do not activate or repress AMP gene transcription in these insect species. Another work tested the transcriptional level of immune genes in Drosophila adults artificially infected with Spiroplasma (strain NSRO) and in uninfected control flies (Hurst et al., 2003). It was observed that the bacteria caused no up-regulation of AMP genes in infected flies, the presence of Spiroplasma did not downregulate the immune response of the fly against heat-killed bacteria and fungal spores, and endosymbiotic bacterial load decreased in flies overexpressing Toll or flies receiving a septic challenge. These results suggest that Spiroplasma bacteria are undetected or they are efficiently restrained by the fly immune system. However, more recently it was reported that titers of the native Spiroplasma strain MSRO are not affected in Drosophila lines carrying null mutations that affect key components of the Toll and Imd immune pathways compared to wild type flies, whereas induction of the systemic immune response by microbial infection or ectopic activation leads to high titers of the endosymbionts (Herren and Lemaitre, 2011). Another study examined the effect of male-killing and non-male-killing Spiroplasma strains (NSRO and NSRO-A, respectively) on the Drosophila immune response (Anbutsu and Fukatsu, 2010). The authors found that neither Spiroplasma strain was able to induce up-regulation of AMP genes in unchallenged flies. Although in flies challenged with dead bacteria or a fungus there was no up-regulation or down-regulation of immune genes by either Spiroplasma strain, flies carrying the male-killing strain showed lower AMP gene transcription levels compared to uninfected controls. Furthermore the male-killing strain was able to proliferate in old Toll gain-of-function mutant flies. These results imply that the close association of Spiroplasma endosymbionts with Drosophila is based on specific strategies that the bacteria employ in order to evade, suppress, and tolerate the host immune response.

The interaction of endosymbiotic bacteria with insect immune recognition genes has previously been established by analyzing the response of the weevil Sitophilus zeamais to its intracellular symbiont, Sitophilus zeamais primary endosymbiont (SPE) (Anselme et al., 2006). It was shown that transcription of peptidoglycan recognition protein gene (PGRP) (ortholog of the Drosophila $P G R P-L B$ ) was increased in the bacteriome and this increase coincides with the release of the endosymbiotic bacteria from the bacteriocytes. In turn, high $P G R P$ gene transcription at the nymphal stage of Sitophilus is accompanied by significant up-regulation of the endosymbiont virulence genes (Dale et al., 2002). Interestingly, SPE injection into the hemolymph of 
Sitophilus triggers a systemic immune activation, whereas persistent SPE infection of bacteriocytes results in the transcriptional induction of the AMP gene Coleoptericin A (ColA) (Anselme et al., 2008). It was recently demonstrated that silencing of ColA by RNAi affects the amount of SPE that exits the bacteriome. This exciting finding implies that this AMP acts not only in response to foreign microorganisms but also regulates bacterial symbiosis in the weevil (Login et al., 2011).

Transcriptional analyses of the interaction between endosymbiotic bacteria and insect host genes have recently been performed in vivo and in vitro. A microarray study on gene transcription of Drosophila melanogaster larval testes has shown that several genes related to humoral and cellular host immune response were up-regulated in the presence of the naturally occurring avirulent w Mel strain of Wolbachia (Zheng et al., 2011), including two AMP genes (Drosomycin and Lysozyme) and a positive regulator in the immune deficiency (Imd) signaling pathway (Kenny). Similarly, transcription profiles of Wolbachia infected and uninfected Drosophila S2 cells revealed the up-regulation of several genes involved in the Imd, Toll, and c-Jun N-terminal kinase (JNK) pathways (Lemaitre and Hoffmann, 2007), such as the NF- $\mathrm{B}$ transcription factors Relish, Dorsal, and dJun (Bohmann et al., 1994; Hetru and Hoffmann, 2009), the sole Drosophila JNK-specific MAPK phosphatase puckered (McEwen and Peifer, 2005), and various AMP genes (Xi et al., 2008). In sharp contrast, transcriptomic characterization of Anopheles gambiae cells transinfected with the Wolbachia strains $w \mathrm{Ri}$ and $w$ AlbB showed down-regulation of over $75 \%$ of the immune related genes involved in pathogen recognition and signaling cascades, and genes encoding effector molecules (Hughes et al., 2011b). Another in vitro study used a silkworm microarray to investigate transcription of Bombyx mori cells infected by Cardinium endosymbiotic bacteria and the Wolbachia strain $w S$ tr, an endosymbiont of the small brown planthopper Laodelphax striatellus (Nakamura et al., 2011). Infection with Cardiniuminduced the transcription of AMP genes, a serine protease gene that participates in the activation of the prophenoloxidase cascade, and two pattern recognition protein genes, while infection with Wolbachia did not activate any immune related genes. Crucially, a recent investigation tested the role of Wolbachia Surface Protein (WSP) in the interaction between the endosymbiont and immune genes in mosquitoes. The authors used cell lines from A. gambiae, which is not a natural host for Wolbachia, and A. albopictus, which is naturally infected with the strain $w \mathrm{AlbB}$, and compared the transcriptional induction of immune genes between the two cell lines (Pinto et al., 2012). The A. gambiae cell-lines showed strong transcriptional up-regulation of certain AMP genes as well as complement-like genes that participate in the elimination of Plasmodium parasites in this mosquito species. In contrast, lower mRNA levels of immune genes were detected in cell-lines from A. albopictus. Interestingly, transcriptional levels in the latter case was dependent on WSP concentration and was mainly restricted to early time points post WSP treatment.

In the parasitoid Asobara tabida, regulation of immune genes by the native Wolbachia strains wAtab1, wAtab2, and wAtab3 was found to be tissue and sex-specific (Kremer et al., 2012).
Numerous upstream genes in the Imd, Toll, JNK, RNAi, and Janus Kinase/Signal Transducer Activator of Transcription (JAK/STAT) pathways were significantly up-regulated in male wasps harboring Wolbachia compared to downstream AMP genes that were mostly down-regulated, while immune genes were transcribed at lower levels in the ovaries of females wasps. The authors concluded that Wolbachia endosymbionts can influence the immune response of the wasp to facilitate persistence and maintain close association with their host. A transcriptomic experiment in the pea aphid A. pisum to test the effect of the facultative symbiont Serratia symbiotica on immune gene transcription in infected and uninfected aphids reported that there were no significant changes in transcription of immune genes. These included recognition genes, AMP genes, thioester-containing protein (TEP) genes, prophenoloxidase, and NO cascade genes (Burke and Moran, 2011). These data support the notion that Serratia endosymbionts are not recognized by the pea aphid immune system. High variation in immune gene transcriptional regulation in the above studies leads us to speculate that endosymbiotic bacteria may adopt different strategies to actively evade the host immune system and promote infection.

It has further been proposed that endosymbiotic bacteria can also interact with the insect cellular immune response and melanization response. It was recently shown that pea aphid lines carrying different secondary symbionts contain variable number of adherent hemocytes (Schmitz et al., 2012). In particular, aphids carrying Hamiltonella defensa or Regiella insecticola symbiotic bacteria contained fewer numbers of hemocytes compared to aphids carrying S. symbiotica or no endosymbionts. Furthermore, B. aphidicola primary endosymbionts were found in phagolysosomes of adherent hemocytes. These data suggest that different endosymbionts can interact in different ways with host immune cells and the identification of endosymbionts in hemocytes might form a mechanism for their successful transmission from the parent aphids to the offspring. Of note, removal of hemocytes from Drosophila through genetic manipulations was not directly linked to variation in Spiroplasma titers in the hemolymph of the fly (Herren and Lemaitre, 2011), and the effect of endosymbionts on melanization levels were found to be higher in Aedes aegypti mosquitoes transinfected with the Wolbachia strain $w$ MelPop compared to uninfected controls (Thomas et al., 2011). Interestingly, control mosquitoes laid darker eggs compared to those deposited by Wolbachia infected individuals, and there were similar levels of dopamine in mosquitoes carrying or lacking Wolbachia endosymbionts. Similar results were also observed in D. melanogaster and D. simulans flies naturally infected with the Wolbachia strains $w \mathrm{Mel}$ and $w$ MelPop. These results further emphasize that endosymbiotic bacteria are able to regulate the intensity of key immune defense mechanisms, like melanization, in their insect hosts.

\section{ENDOSYMBIONTS AND THE INSECT IMMUNE RESPONSE TO PATHOGENS \\ EFFECT OF ENDOSYMBIONTS ON BACTERIAL INFECTIONS}

The impact of Wolbachia and Spiroplasma endosymbionts to insect antibacterial immune responses has recently drawn the attention of insect immunologists. A comprehensive study has 
lately investigated the involvement of Spiroplasma in the response against Gram-positive and Gram-negative bacterial pathogens (Herren and Lemaitre, 2011). This work revealed that flies carrying the Spiroplasma strain MSRO, which naturally infects D. melanogaster, were more susceptible to septic injury with the Gram-negative bacteria Erwinia carotovora and Enterobacter cloacae, but not with the Gram-positive bacterium Enterococcus faecalis or the fungus Beauveria bassiana, compared to flies lacking the endosymbiont. These results strongly suggest that Spiroplasma endosymbionts are potentially able to alter the sensitivity of flies to some bacterial pathogens. Furthermore, there was no difference in Toll or Imd pathway activation between flies containing or lacking Spiroplasma upon infection with the bacteria Micrococcus luteus (Gram-positive) or E. carotovora (Gramnegative), respectively. Surprisingly, infection of wild type flies with the plant pathogenic bacterium Spiroplasma citri failed to activate the immune system, leading to bacterial proliferation in the hemolymph and death of the flies. In addition, wild type and immune mutants infected by $S$. citri showed no differences in their survival rates following infection with this bacterium. The ability of S. citri to kill flies is probably due to the fact that these bacteria are not detected by the Drosophila immune system.

Challenge of Wolbachia-infected D. melanogaster and D. simulans flies (strains $w \mathrm{Au}, w \mathrm{Ri}, w \mathrm{No}, w \mathrm{Ha}$, and $w \mathrm{MelCS}$, respectively), with three gram-negative bacterial pathogens (Pseudomonas aeruginosa PA01, Serratia marcescens, and E. carotovora) did not affect their survival ability compared to Wolbachia-free control flies (Wong et al., 2011). In addition, no differences in pathogen load and transcription of AMP genes was found between the flies carrying or lacking Wolbachia endosymbionts. These results suggest that native Wolbachia endosymbionts do not confer antibacterial immune priming in Drosophila. Apart from studies testing the effect of Wolbachia on survival of flies infected with extracellular bacteria, similar research has lately been carried out using intracellular bacterial pathogens. Experiments involving infection of D. melanogaster flies naturally infected with Wolbachia (probably strain $w \mathrm{Mel}$ ) with two intracellular bacterial pathogens, Salmonella typhimurium and Listeria monocytogenes, and an extracellular pathogen, Providencia rettgeri, showed no differences in pathogen load between the two types of flies (Rottschaefer and Lazzaro, 2012) (Figure 1). These results indicate that the presence of Wolbachia in Drosophila does not affect replication of these intracellular pathogens in the fly. Similar studies have also expanded to important insect vectors of human diseases. In particular, A. aegypti mosquitoes transinfected with the Wolbachia strain wMelPop showed increased resistance to infection with E. carotovora bacteria (Kambris et al., 2009) (Figure 2). This effect was probably due to up-regulation of immune effectors, such as AMP molecules, in the Wolbachia-infected mosquitoes. Although these results imply that Wolbachia can potentially protect against bacterial infections, additional tests with other bacterial pathogens and knockdown studies would be extremely useful for verifying this hypothesis. Furthermore, the results in Drosophila and mosquitoes indicate that native and experimentally introduced
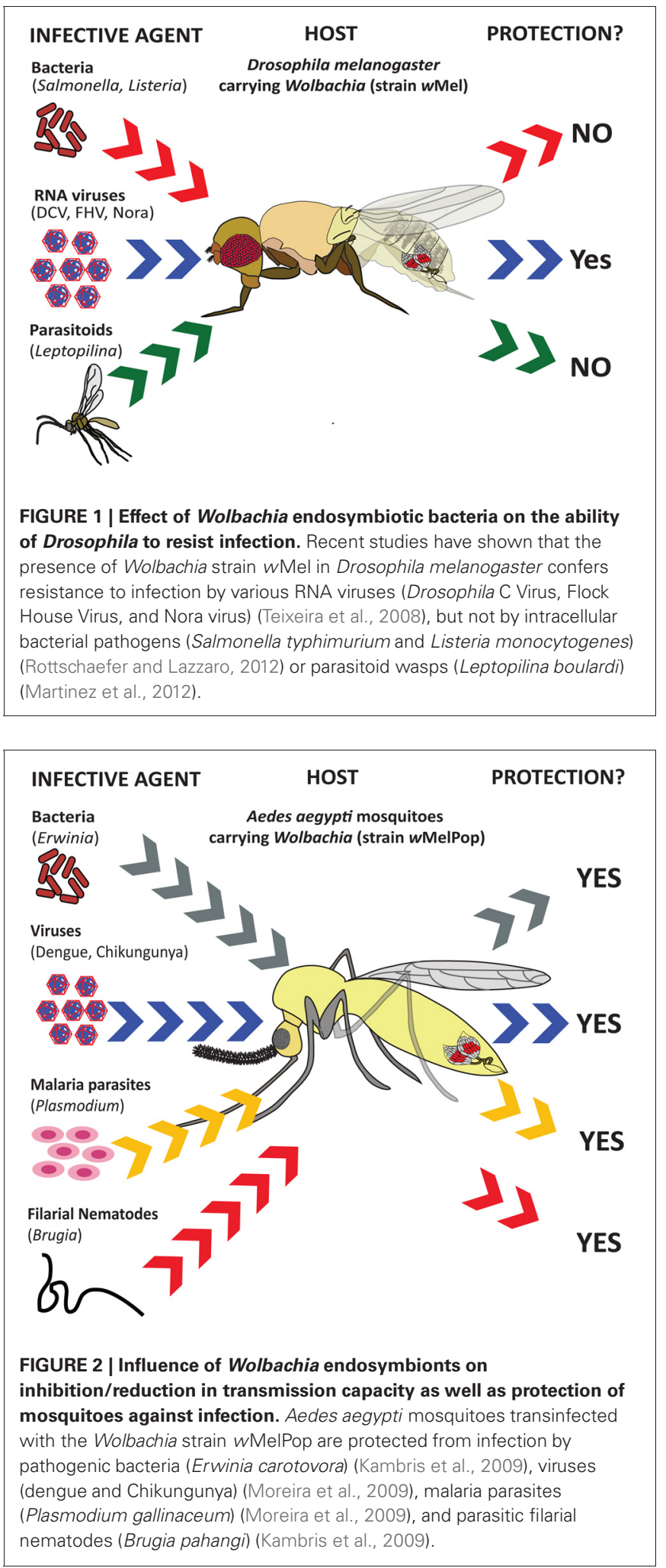

Wolbachia endosymbionts modulate immune responses in distinct manners in different hosts.

The immune response of tsetse flies to infection with symbiotic and foreign bacteria was previously examined (Weiss 
et al., 2008). The authors infected tsetse flies with E. coli and S. glossinidius bacteria and tested the transcriptional levels of several immune related genes in the host. They found that infection with virulent E. coli K12 bacteria failed to induce transcriptional activation of immune genes whereas infection with non-virulent wild type $S$. glossinidius bacteria resulted in upregulation of several immune genes. It was also shown that $E$. coli was cleared from the hemolymph, probably because these bacteria are unable to manipulate host immune responses to their advantage, and S. glossinidius persisted, probably due to increased resistance of the bacteria to host immune effector molecules. The same research group recently performed elegant experiments using tsetse flies lacking their endogenous symbiotic microbes (aposymbiotic flies) and showed that they were sensitive to systemic infection with $E$. coli bacteria (Weiss et al., 2012). This sensitivity was attributed to down-regulation of numerous immune genes and in particular those associated with the cellular immune response as well as to the absence of phagocytic hemocytes. However, transfer of hemocytes from wild type to aposymbiotic flies prior to challenge with E. coli reversed the susceptible phenotype. The resistant phenotype was also observed when aposymbiotic offspring of symbiont-cured female flies were fed on diet supplemented with cell extracts from tsetse's obligate symbiont, Wigglesworthia. These findings suggest that Wigglesworthia bacteria participate actively in the development and activation of the immune system of their host.

Transcriptional analysis of immune genes that are regulated in Sitophilus larvae carrying or lacking SPE endosymbiotic bacteria upon infection with $E$. coli bacteria provided further insight into the role of endosymbionts in regulating the host immune response (Vigneron et al., 2012). Surprisingly, it was reported that larvae without the endosymbionts exhibited stronger induction of several immune related genes, including AMP genes and canonical components of immune signaling pathways, compared to symbiotic insects. This is probably due to manipulation of host immune gene expression by the endosymbiont or due to differential immune activity by the host in order to maintain the endosymbionts in the bacteriome and trigger a response to foreign microbes.

\section{EFFECT OF ENDOSYMBIOTIC BACTERIA ON VIRAL INFECTIONS}

There has been a dramatic increase in the number of publications on the interaction between endosymbiotic bacteria and the insect immune response to viral pathogens. It was originally shown that D. melanogaster wild type adult flies naturally infected with the Wolbachia strains $w \mathrm{MelCS}$ and $w$ MelPop survive longer an infection by the RNA viruses Drosophila C Virus (DCV), Flock House Virus (FHV) and Cricket Paralysis Virus (CrPV) (Hedges et al., 2008). A concurrent study verified and extended these results by reporting increased resistance of Wolbachia w $\mathrm{Mel}$ infected D. melanogaster flies to Nora virus (RNA virus), but not to Insect Virus 6 (DNA virus), and reduced viral burden in flies carrying the endosymbionts (Teixeira et al., 2008) (Figure 1). The Wolbachia-mediated protection against DCV and FHV is independent of the small interfering RNA pathway that inhibits viral replication by sequence-specific degradation of viral RNA (Ghildiyal and Zamore, 2009). In particular, it was shown that Dicer-2, Argonaute2, and r2d2 loss-of-function mutant flies naturally carrying Wolbachia wMel endosymbionts succumbed at a far slower rate to infection by DCV and FHV compared to mutants free of the endosymbionts (Hedges et al., 2012).

The antiviral effect of Wolbachia is not restricted to D. melanogaster flies only. It has been shown that $D$. simulans flies naturally harboring Wolbachia endosymbionts can also be protected against DCV and FHV infection, but the protection varies with different strains of Wolbachia (Osborne et al., 2009). The three Wolbachia strains that gave strong avtiviral protection in $D$. simulans were $w \mathrm{Mel}, w \mathrm{Ri}$ and $w \mathrm{Au}$, but not the strains $w \mathrm{Ha}$ and $w$ No. However, increased protection to DCV does not necessarily reflect lower levels of viral load in the Wolbachia infected flies. The authors further found increased density of the three Wolbachia strains that confer antiviral protection to D. simulans. Interestingly, a male-killing native Wolbachia strain does not protect $D$. bifasciata flies from DCV and FHV (Longdon et al., 2012). This probably indicates genetic differences between the male-killing Wolbachia strain and those that offer antiviral protection to their hosts. Recent data also demonstrate that the presence of $w \mathrm{Mel}$ related Wolbachia in D. melanogaster is beneficial because the endosymbiont is able to inhibit replication of bluetongue virus, which otherwise replicates efficiently in flies free of Wolbachia (Shaw et al., 2012). However, the genetic basis of this effect is currently unclear.

Wolbachia endosymbionts can also have detrimental effects to their hosts. Recent laboratory and field investigations suggest that the Wolbachia strain wExe1,wExe2, and wExe3 naturally found in larvae of the African armyworm, Spodoptera exempta, significantly enhances the mortality caused by a double-stranded DNA baculovirus (Graham et al., 2012). The authors speculate that this could be due to the eco-evolutionary dynamics of the interaction between Wolbachia and the baculovirus, which are both obligate intracellular symbionts in the armyworm but they use distinct modes of transmission.

Several studies have also examined the interaction between Wolbachia infection in mosquitoes and their vector competence for important mammalian viral pathogens. It was originally found that introduction of the wMelPop-CLA Wolbachia strain into $A$. aegypti reduces the ability of two arboviruses (dengue virus and Chikungunya virus) and the avian malaria parasite (Plasmodium gallinaceum) to establish infection in the mosquito (Moreira et al., 2009) (Figure 2). The resistant phenotype was attributed to the approximately 100 -fold up-regulation of genes encoding immune effectors, such as the AMP Cecropin and Defensin, TEP proteins, and C-type lectins, in Wolbachia infected mosquitoes compared to uninfected controls or competition for important host cell components. Of note, transinfection of old $A$. aegypti mosquitoes with the wMelPop strain of Wolbachia results in changes in virulence that lead to behavioral effects that in turn reduce blood-feeding efficiency (Turley et al., 2009). A. aegypti mosquitoes transinfected with Wolbachia were also found to suppress dengue virus replication, dissemination, and transmission compared to uninfected controls (Bian et al., 2010). These effects were associated with higher transcription of Cecropin and Defensin, as well as up-regulation of the Toll pathway genes Rel1, Spz1A, and GNBP1 in Wolbachia 
infected mosquitoes, but not in those lacking the endosymbionts. Wolbachia infection in A. aegypti causes oxidative stress and high levels of ROS. It was recently shown that Toll pathway activation in A. aegypti transinfected with the Wolbachia strain $w$ AlbB is also linked to the up-regulation of genes that control reductionoxidation (redox) reactions (Pan et al., 2012). Transcriptional activation of redox related genes are important for preventing adverse effects of oxidative stress in the mosquito. These results imply that Wolbachia is capable of regulating the Toll pathway in A. aegypti; this might constitute a mechanism for controlling dengue infection in mosquitoes.

Certain Wolbachia strains ( $w \mathrm{Mel}$ and $w$ MelPop-CLA) in D. melanogaster have been introduced into A. aegypti mosquitoes where they have been found to increase the transcriptional levels of melanization genes as well as AMP and Toll related genes (Rancès et al., 2012). Up-regulation of immune genes in Wolbachia transinfected mosquitoes probably leads to overreplication of the bacteria in the new host that could result in immune priming. However, it was observed that although dengue accumulation is reduced in both $D$. melanogaster and A. aegypti infected with Wolbachia, there is no up-regulation of the same genes in the fly. These findings denote that transcriptional induction of the specific immune genes is probably not required to reduce dengue accumulation in the mosquito host. The lack of up-regulation of these immune genes by native Wolbachia endosymbionts in the fly with simultaneous suppression of dengue also suggests that reduction of virus accumulation in Drosophila is likely due to host physiological or metabolic responses. Such responses could interfere with pathogen replication and therefore alter the outcome of infection. Given that $w \mathrm{Mel}$ and $w$ MelPop differentially regulate the expression of immune genes in the fly, it is further possible that distinct physiological/metabolic changes are caused by each Wolbachia strain. Another possibility could be that native Wolbachia strains in Drosophila have different needs for nutritional supplies or competition for resources between the endosymbionts and the pathogen could also modify the infection status and efficiency of the host immune response. These various possibilities need to be explored in future studies.

Inhibition of dengue replication in A. aegypti mosquitoes has also been confirmed in vitro using $A$. aegypti cell lines transinfected with the wMelPop-CLA Wolbachia strain (Frentiu et al., 2010). These experiments showed that mosquito cells proliferate at slower rates in the presence of Wolbachia and viral protection correlates with the density of the endosymbiotic bacteria contained in each cell. Interestingly, the Wolbachia-mediated protective effect against dengue was not observed in Aedes albopictus mosquitoes that naturally carry the $w \mathrm{AlbA}$ and $w \mathrm{AlbB}$ strains of the endosymbiont ( $\mathrm{Lu}$ et al., 2012). Using in vivo and in vitro assays the authors showed that lack of A. albopictus resistance to dengue virus depends strongly on Wolbachia density that is extremely low in midgut, fat body and salivary glands compared to A. aegypti. Another study also recently reported that although A. albopictus transmits dengue in the presence of its naturally occurring Wolbachia endosymbionts, transmission of the virus is blocked in $w \mathrm{Mel}$ transinfected mosquitoes in the absence of significant immune gene up-regulation (Blagrove et al., 2012).
These results suggest that dengue transmission inhibition by A. albopictus appears to be limited to lines harboring specific exogenous strains of Wolbachia. In the same mosquito species, replication of Chikungunya virus was increased after 4 days of infection with simultaneous decrease in density of naturally occurring Wolbachia endosymbionts (strains $w \mathrm{AlbA}$ and $w \mathrm{AlbB}$ ) (Mousson et al., 2010). This phenotype was observed in whole mosquitoes as well as in the midgut and salivary gland tissues that play a key role in viral transmission. Strikingly, there was high variation in viral load in A. albopictus free of Wolbachia, which indicates that the endosymbiont is not probably required for Chikungunya replication in this mosquito. A similar recent study in A. albopictus has also confirmed that Wolbachia numbers decrease with infection by Chikungunya (Zouache et al., 2012). In another mosquito vector, the southern house mosquito Culex quinquefasciatus, natural Wolbachia infection improves resistance to West Nile virus by lowering viral titers and transmission during feeding compared to mosquitoes without Wolbachia (Glaser and Meola, 2010). Interestingly, the authors reported that the protective effect conferred by Wolbachia was more pronounced in Drosophila flies infected with the virus compared to C. quinquefasciatus mosquitoes, but the molecular basis of this difference was not investigated.

\section{EFFECT OF ENDOSYMBIOTIC BACTERIA ON PARASITIC INFECTIONS}

Recent efforts to examine the potential role of endosymbiotic bacteria in the insect immune response to parasites have mainly focused on Wolbachia and Wigglesworthia. A study involving Wolbachia infected A. gambiae cell-lines and intrathoracic inoculation of endosymbionts into adult mosquitoes showed that the presence of the endosymbiont increased the transcription of selected immune genes (e.g., TEP1) compared to control treatments (Kambris et al., 2010). Gene up-regulation resulted in lower number of Plasmodium berghei oocysts in the Wolbachia-infected mosquitoes. Furthermore, silencing of TEP1 in mosquitoes containing the endosymbiont increased oocyst numbers, which demonstrates that Wolbachia-induced over-transcription is an important factor for the inhibition of the parasite. Similar experiments also showed reduced numbers of Brugia pahangi parasites in A. aegypti somatically infected with Wolbachia.

It is of particular interest to note that not all Wolbachia strains confer pathogen resistance to their hosts. It was recently observed that the Wolbachia strain wAlbB from A. albopictus mosquitoes does not affect the survival of somatically infected A. gambiae mosquitoes after feeding on $P$. berghei infected mice, whereas the strain $w$ MelPop from $D$. melanogaster reduces the survival of mosquitoes after blood feeding (Hughes et al., 2012). In addition, strain $w$ AlbB increases the numbers of $P$. berghei oocysts in the A. gambiae midgut but strain wMelPop decreases oocyst numbers, while $w$ MelPop reaches higher densities than $w$ AlbB in infected mosquitoes. The authors conclude that these phenotypic differences in the anti-Plasmodium response of A. gambiae carrying different strains of Wolbachia may reflect the effect of the endosymbiont on the mosquito immune response. In a similar study, wMelPop strain was found in several tissues throughout the mosquito, but it was not detected in the gut and ovaries 
(Hughes et al., 2011a). It was further shown that wMelPop and $w$ AlbB strains can substantially reduce Plasmodium falciparum oocysts in the A. gambiae gut, Wolbachia infection differentially regulates several immune genes in the mosquito, and that both strains efficiently replicate in their host. The authors propose that these findings could be useful for employing Wolbachia as a means to reduce transmission of Plasmodium parasites. In agreement with previous results, two strains of Wolbachia pipientis, $w \operatorname{Pip}(\mathrm{Sl})$ and $w \operatorname{Pip}(\mathrm{Mc})$, naturally found in Culex pipiens were also shown to confer protection to mosquitoes following infection with Plasmodium relictum parasites (Zélé et al., 2012).

Wolbachia endosymbionts in mosquitoes can also affect the development of other important human parasites, such as filarial nematodes. In particular, whole-genome microarray experiments demonstrated that transinfection of $A$. aegypti with the $w$ MelPop strain of Wolbachia leads to up-regulation of a considerable number of genes (especially Cecropins and other AMP or effector genes) compared to uninfected mosquitoes, which may be responsible for the inhibitory effect on the development of B. pahangi parasites (Kambris et al., 2009) (Figure 2).

In Drosophila, it was recently nicely demonstrated that mushroom-feeding D. neotestacea flies harboring Spiroplasma endosymbionts show increased tolerance to its natural nematode parasite Howardula aoronymphium in both the wild and the lab (Jaenike et al., 2010). The authors showed that the presence of Spiroplasma can rescue the fertility of female $D$. neotestacea flies parasitized with the nematodes. This rescue is not observed in Wolbachia-infected or endosymbiont-free flies parasitized with the nematodes. This tolerant phenotype was attributed to an unknown mechanism that reduces the growth and reproductive ability of the worms in the Spiroplasma-infected flies. It was further shown that numbers of Howardula nematodes decreases in D. neotestacea populations carrying Spiroplasma, but spread in populations lacking the endosymbiont (Jaenike and Brekke, 2010). These results imply that Spiroplasma endosymbionts have a major influence on the population dynamics of the host and its natural parasite.

In tsetse flies, the absence of native Wigglesworthia endosymbionts was originally shown to increase the numbers of trypanosome parasites and therefore significantly affected the vectorial competence of the flies (Pais et al., 2008). Protection of tsetse flies to trypanosome parasites has been attributed to the level of PGRP-LB gene transcription (Wang et al., 2009). Wigglesworthia induces the transcription of PGRP-LB in the bacteriome of the tsetse fly compared to flies lacking the endosymbiont. Interestingly, challenge of wild type or relish-silenced flies with E. coli bacteria does not affect PGRP-LB mRNA levels in the bacteriome, which suggests that transcription of PGRP-LB in the bacteriome is regulated by the endosymbiont. However, the molecular mechanism responsible for this effect is currently unknown. Transcription of PGRP-LB also affects symbiotic homeostasis since density of Wigglesworthia endosymbionts decreases when PGRP-LB is silenced. Furthermore, PGRP-LB transcription is important for resistance to trypanosome infection and transmission of the parasite. A more recent work has shown that $P G R P-L B$ prevents immune activation and is therefore a crucial factor for protecting Wigglesworthia from detrimental effects on the host (Wang and Aksoy, 2012). In addition, tsetse larvae from $R G R P$ - $L B$-silenced mothers produce adults with reduced immune capacity. Finally, the authors nicely demonstrate that expression of recombinant PGRP-LB exhibit bactericidal and trypanocidal activity that potentially mediate the immune response of tsetse to infections.

\section{EFFECT OF ENDOSYMBIOTIC BACTERIA ON PARASITOID ATTACKS}

The effect of Wolbachia and Spiroplasma endosymbionts on the immune response of Drosophila against parasitoids has also been a subject for investigation. In a previous study, the encapsulation ability of $D$. simulans larvae naturally infected with Wolbachia was estimated upon infection with the parasitoid wasp Leptopilina heterotoma (Fytrou et al., 2006). It was shown that larvae carrying Wolbachia were less able to encapsulate parasitoid eggs compared to larvae lacking the endosymbiont, which suggests that the presence of Wolbachia in Drosophila suppresses the host cellular immune response against parasitism. Crucially, the mechanistic basis of this effect was not investigated. In addition, the authors observed that the presence of Wolbachia has no effect on the survival of male adult flies infected by the entomopathogenic fungus $B$. bassiana. However, a similar recent work concluded that native Wolbachia strains in D. melanogaster ( $w \mathrm{Mel}$ and $w$ MelPop) and $D$. simulans ( $w$ Ri) larvae have no influence on the encapsulation of parasitoid eggs, although there is a minor decrease in parasitoid development in flies infected by the endosymbiont (Martinez et al., 2012) (Figure 1). In contrast to the previous results, Spiroplasma endosymbionts increase the ability of Drosophila hydei to survive infection by Leptopilina wasps (Xie et al., 2010). This protective effect was later attributed to the high reproductive capacity of Spiroplasma-infected flies under conditions of parasitoid challenge (Xie et al., 2011). Finally, it was recently shown that Wolbachia endosymbionts (strain ST 306) in the parasitoid wasp Leptopilina victoria do not affect encapsulation of its eggs by various Drosophila host species (Gueguen et al., 2012).

\section{CONCLUSIONS AND FUTURE PROSPECTS}

Despite impressive advances in the broad field of insect innate immunity, our understanding of the role of endosymbiotic bacteria in the host immune response to pathogenic infections remains incomplete. Previous and recent studies have started to determine the phenotypic response of various insects carrying endosymbionts to infection by bacterial and viral pathogens as well as parasites. These studies have substantially improved our understanding of the complex interactions between insects, their endosymbiotic bacteria and pathogenic organisms in the infection and host immunity processes. It will further be of particular interest to test the immune response of insects with or without endosymbionts to infection by entomopathogenic fungi, as there is currently clear conflict within the literature on the effect of Wolbachia on fungal infections (Fytrou et al., 2006; Panteleev et al., 2007). Another major challenge is to gain a substantially more detailed and comprehensive grasp on how exactly endosymbiotic bacteria regulate insect immune defense mechanisms against pathogens and parasites. The identity of these mechanisms can be vigorously investigated via genetic, 
molecular and genome-wide transcriptome analyses in various insect models. Such studies will lay the foundation for exploring whether endosymbiotic bacteria also cause functional changes in the immune system of vertebrate animals. An additional challenge is to characterize the interplay between different endosymbionts, such as Wolbachia and Spiroplasma, co-existing in an insect host and efficiency of the immune function. It will also be important to elucidate the precise mechanisms employed by endosymbiotic bacteria to modulate immune signaling in insects. Similar research will undoutedly reveal the relative contribution of endosymbiotic bacteria to the overall host immune response against various classes of pathogenic organisms.

From the practical point of view, the recent discovery that the presence of Wolbachia endosymbionts in mosquitoes has a direct effect on insect sensitivity to pathogenic infections has attracted the attention of scientists for the development of novel approaches for the control of human diseases (Hancock

\section{REFERENCES}

Aksoy, S., and Rio, R. V. (2005). Interactions among multiple genomes: tsetse, its symbionts and trypanosomes. Insect Biochem. Mol. Biol. 35, 691-698.

Anbutsu, H., and Fukatsu, T. (2010). Evasion, suppression and tolerance of Drosophila innate immunity by a male-killing Spiroplasma endosymbiont. Insect Mol. Biol. 19, 481-488.

Anselme, C., Pérez-Brocal, V., Vallier, A., Vincent-Monegat, C., Charif, D., Latorre, A., et al. (2008). Identification of the weevil immune genes and their expression in the bacteriome tissue. BMC Biol. 6:43. doi: 10.1186/1741-7007-6-43

Anselme, C., Vallier, A., Balmand, S., Fauvarque, M.-O., and Heddi, A. (2006). Host PGRP gene expression and bacterial release in endosymbiosis of the weevil Sitophilus zeamais. Appl. Environ. Microbiol. 72, 6766-6772.

Balmand, S., Lohs, C., Aksoy, S., and Heddi, A. (2012). Tissue distribution and transmission routes for the tsetse fly endosymbionts. J. Invertebr. Pathol. doi: 10.1016/ j.jip.2012.04.002. [Epub ahead of print].

Bian, G., Xu, Y., Lu, P., Xie, Y., and Xi, Z. (2010). The endosymbiotic bacterium Wolbachia induces resistance to dengue virus in Aedes aegypti. PLoS Pathog. 6:e1000833. doi: 10.1371/journal.ppat.1000833

Blagrove, M. S., Arias-Goeta, C., Failloux, A. B., and Sinkins, S. P. (2012). Wolbachia strain wMel induces cytoplasmic incompatibility and blocks dengue transmission in Aedes albopictus. Proc. Natl. Acad. Sci. U.S.A. 109, 205-260.
Bohmann, D., Ellis, M. C., Staszewski, L. M., and Mlodzik, M. (1994). Drosophila Jun mediates Ras-dependent photoreceptor determination. Cell 78, 973-986.

Bourtzis, K., Pettigrew, M. M., and O'Neill, S. L. (2000). Wolbachia neither induces nor suppresses transcripts encoding antimicrobial peptides. Insect Mol. Biol. 9, 635-639.

Bright, M., and Bulgheresi, S. (2010). A complex journey: transmission of microbial symbionts. Nat. Rev. Microbiol. 8, 218-230.

Burke, G. R., and Moran, N. A. (2011). Responses of the pea aphid transcriptome to infection by facultative symbionts. Insect Mol. Biol. 20, 357-365.

Chambers, M. C., and Schneider, D. S. (2012). Pioneering immunology: insect style. Curr. Opin. Immunol. 24, 10-14.

Dale, C., Plague, G. R., Wang, B., Ochman, H., and Moran, N. A. (2002). Type III secretion systems and the evolution of mutualistic endosymbiosis. Proc. Natl. Acad. Sci. U.S.A. 99, 12397-12402.

Davis, M. M., and Engström, Y. (2012). Immune response in the barrier epithelia: lessons from the fruit fly Drosophila melanogaster. J. Innate Immun. 4, 273-283.

Dobson, S. L., Bourtzis, K., Braig, H. R., Jones, B. F., Zhou, W., Rousset, F., et al. (1999). Wolbachia infections are distributed throughout insect somatic and germ line tissues. Insect Biochem. Mol. Biol. 29, 153-160.

Douglas, A. E. (2011). Lessons from studying insect symbiosis. Cell Host Microbe 10, 359-367.

Eleftherianos, I., and Revenis, C. (2011). Role and importance of

et al., 2011). For example, it was recently demostrated that Wolbachia introduced into A. aegypti resulted in successful invasion of natural populations of mosquitoes (Hoffmann et al., 2011; Walker et al., 2011). Such approaches can be potentially implemented in field practices for the effective disruption of dengue transmission by mosquitoes. Finally, a better understanding of insect-symbiont-pathogen interactions will lead to more efficient management strategies, particularly those involving integrated and biological control tactics, those seeking to reduce reliance on broad-spectrum insecticides, and those involving the better deployment of insect-specific pathogens.

\section{ACKNOWLEDGMENTS}

We apologize to those whose work is not cited due to space limitations. We thank members of the Biological Sciences Department at George Washington University (GWU) for critical reading of the manuscript and the Columbian College of Arts and Sciences at GWU for funding.

phenoloxidase in insect hemostasis. J. Innate Immun. 3, 28-33.

Feldhaar, H., and Gross, R. (2009). Insects as hosts for mutualistic bacteria. Int. J. Med. Microbiol. 299, 1-8.

Frentiu, F. D., Robinson, J., Young, P. R., McGraw, E. A., and O'Neill, S. L. (2010). Wolbachia-mediated resistance to dengue virus infection and death at the cellular level. PLoS ONE 5:e13398. doi: 10.1371/ journal.pone.0013398

Fytrou, A., Schofield, P. G., Kraaijeveld, A. R., and Hubbard, S. F. (2006) Wolbachia infection suppresses both host defence and parasitoid counter-defence. Proc. Biol. Sci. 273, 791-796.

Ganesan, S., Aggarwal, K., Paquette, N., and Silverman, N. (2011). NF$\mathrm{kB} /$ Rel proteins and the humoral immune responses of Drosophila melanogaster. Curr. Top. Microbiol. Immunol. 349, 25-60.

Ghildiyal, M., and Zamore, P. D. (2009). Small silencing RNAs: an expanding universe. Nat. Rev. Genet. 10, 94-108.

Glaser, R. L., and Meola, M. A. (2010). The native Wolbachia endosymbionts of Drosophila melanogaster and Culex quiquefasciatus increase host resistance to West Nile virus infection. PLoS ONE 5:e11977. doi: 10.1371/ journal.pone.0011977

Graham, R. I., Grzywacz, D., Mushobozi, W. L., and Wilson, K. (2012). Wolbachia in a major African crop pest increases susceptibility to viral disease rather than protects. Ecol. Lett. 15, 993-1000.

Gross, R., Vavre, F., Heddi, A., Hurst, G. D., Zchori-Fein, E., and Bourtzis,
K. (2009). Immunity and symbiosis. Mol. Microbiol. 73, 751-759.

Gueguen, G., Onemola, B., and Govind, S. (2012). Association of a new Wolbachia strain with, and its effects on, Leptopilina victoriae, a virulent wasp parasitic to Drosophila spp. Appl. Environ. Microbiol. 78, 5962-5966.

Hancock, P. A., Sinkins, S. P., and Godfray, H. C. (2011). Strategies for introducing Wolbachia to reduce transmission of mosquito-borne diseases. PLoS Negl. Trop. Dis. 5:e1024. doi: 10.1371/journal.pntd.0001024

Haselkorn, T. S. (2010). The Spiroplasma heritable bacterial endosymbiont of Drosophila. Fly 4 , 80-87.

Hedges, L. M., Brownlie, J. C., O’Neill, S. L., and Johnson, K. N. (2008). Wolbachia and virus protection in insects. Science 322:702. doi: 10.1126/science. 1162418

Hedges, L. M., Yamada, R., O’Neill, S. L., and Johnson, K. M. (2012). The small interfering RNA pathway is not essential for Wolbachiamediated antiviral protection in Drosophila melanogaster. Appl. Environ. Microbiol. 78, 6773-6776.

Herren, J. K., and Lemaitre, B. (2011). Spiroplasma and host immunity: activation of humoral immune responses increases endosymbiont load and susceptibility to certain Gram-negative bacterial pathogens in Drosophila melanogaster. Cell. Microbiol. 13, 1385-1396.

Hetru, C., and Hoffmann, J. A. (2009). NF-kappaB in the immune response of Drosophila. Cold Spring Harb. 
Perspect. Biol. 1:a000232. doi: 10.1101/cshperspect.a000232

Hoffmann, A. A., Montgomery, B. L., Popovici, J., Iturbe-Ormaetxe, I., Johnson, P. H., Muzzi, F., et al. (2011). Successful establishment of Wolbachia in Aedes populations to suppress dengue transmission. Nature 476, 454-457.

Hughes, G. L., Koga, R., Xue, P., Fukatsu, T., and Rasgon, J. L. (2011a). Wolbachia infections are virulent and inhibit the human malaria parasite Plasmodium falciparum in Anopheles gambiae. PLoS Pathog. 7:e1002043. doi: 10.1371/journal.ppat.1002043

Hughes, G. L., Ren, X., Ramirez, J. L., Sakamoto, J. M., Bailey, J. A., Jedlicka, A. E., et al. (2011b). Wolbachia infections in Anopheles gambiae cells: transcriptomic characterization of novel host-symbiont interaction. PLoS Pathog. 7:e1001296. doi: 10.1371/journal.ppat.1001296

Hughes, G. L., Vega-Rodriguez, J., Xue, P., and Rasgon, J. L. (2012). Wolbachia strain $w$ AlbB enhances infection by the rodent malaria parasite Plasmodium berghei in Anopheles gambiae mosquitoes. Appl. Environ. Microbiol. 78, 1491-1495.

Hurst, G. D. D., Anbutsu, H., Kutsukake, M., and Fukatsu, T. (2003). Hidden from the host: Spiroplasma bacteria infecting Drosophila do not cause an immune response, but are suppressed by ectopic immune activation. Insect Mol. Biol. 12, 93-97.

Jaenike, J., and Brekke, T. D. (2010). Defensive endosymbionts: a cryptic trophic level in community ecology. Ecol. Lett. 14, 150-155.

Jaenike, J., Unckless, R., Cockburn, S. N., Boelio, L. M., and Perlman, S. J. (2010). Adaptation via symbiosis: recent spread of a Drosophila defensive symbiont. Science 329, 212-215.

Kambris, Z., Blagborough, A. M., Pinto, S. B., Blagrove, M. S., Godfray, H. C., Sinden, R. E., et al. (2010). Wolbachia stimulates immune gene expression and inhibits Plasmodium development in Anopheles gambiae. PLoS Pathog. 6:e1001143. doi: 10.1371/journal.ppat.1001143

Kambris, Z., Cook, P. E., Phuc, H. K., and Sinkins, S. P. (2009). Immune activation by life-shortening Wolbachia and reduced filarial competence in mosquitoes. Science 326, 134-136.

Kemp, C., and Imler, J. L. (2009). Antiviral immunity in drosophila. Curr. Opin. Immunol. 21, 3-9.
Kikuchi, Y. (2009). Endosymbiotic bacteria in insects: their diversity and culturability. Microbes Environ. 24, 195-204.

Kremer, N., Charif, D., Henri, H., Gavory, F., Wincker, P., Mavingui, P., et al. (2012). Influence of Wolbachia on host gene expression in an obligatory symbiosis. BMC Microbiol. 12:S7. doi: 10.1186/1471-2180-12S1-S7

Lemaitre, B., and Hoffmann, J. (2007). The host defense of Drosophila melanogaster. Annu. Rev. Immunol. 25, 697-743.

Login, F. H., Balmand, S., Valiier, A., Vincent-Monegat, C., Vigneron, A., Weiss-Gayet, M., et al. (2011) Antimicrobial peptides keep insect endosymbionts under control. Science 334, 362-365.

Longdon, B., Fabian, D. K., Hurst, G. D., and Jiggins, F. M. (2012). Malekilling Wolbachia do not protect Drosophila bifasciata against viral infection. BMC Microbiol. 12:S8. doi: 10.1186/1471-2180-12-S1-S8

Lu, P., Bian, G., Pan, X., and Xi, Z. (2012). Wolbachia induces density-dependent inhibition to dengue virus in mosquito cells. PLoS Negl. Trop. Dis. 6:e1754. doi: 10.1371/journal.pntd.0001754

Marmaras, V. J., and Lampropoulou, M. (2009). Regulators and signalling in insect haemocyte immunity. Cell. Signal. 21, 186-195.

Martinez, J., Duplouy, A., Woolfit, M., Vavre, F., O'Neill, S. L., and Varaldi, J. (2012). Influence of the virus LbFV and of Wolbachia in a host-parasitoid interaction. PLoS ONE 7:e35081. doi: 10.1371/journal.pone.0035081

Mateos, M., Castrezana, S. J., Nankivell, B. J., Estes, A. M., Markow, T. A., and Moran, N. A. (2006). Heritable endosymbionts of Drosophila. Genetics 174, 363-376.

Matthew, C. Z., Darby, A. C., Young, S. A., Hume, L. H., and Welburn, S. C. (2005). The rapid isolation and growth dynamics of the tsetse symbiont Sodalis glossinidius. FEMS Microbiol. Lett. 248, 69-74.

McEwen, D. G., and Peifer, M. (2005). Puckered, a Drosophila MAPK phosphatase, ensures cell viability by antagonizing JNK-induced apoptosis. Development 132, 3935-3946.

Moreira, L. A., Iturbe-Ormaetxe, I., Jeffery, J. A., Lu, G., Pyke, A. T., Hedges, L. M., et al. (2009). A Wolbachia symbiont in Aedes aegypti limits infection with dengue, Chikungunya, and Plasmodium. Cell 139, 1268-1278.
Mousson, L., Martin, E., Zouache, K., Madec, Y., Mavinqui, P., and Failloux, A. B. (2010). Wolbachia modulates Chikungunya replication in Aedes albopictus. Mol. Ecol. 19, 1953-1964.

Nakamura, Y., Gotoh, T., Imanishi, S., Mita, K., Kurtti, T. J., and Noda, H. (2011). Differentially expressed genes in silkworm cell cultures in response to infection by Wolbachia and Cardinium endosymbionts. Insect Mol. Biol. 20, 279-289.

Oliver, K. M., Degnan, P. H., Burke, G. R., and Moran, N. A. (2010). Facultative symbionts in aphids and the horizontal transfer of ecologically important traits. Annu. Rev. Entomol. 55, 247-266.

Osborne, S. E., Leong, Y. S., O’Neill, S. L., and Johnson, K. N. (2009). Variation in antiviral protection mediated by different Wolbachia strains in Drosophila simulans. PLoS Pathog. 5:e1000656. doi: 10.1371/journal.ppat.1000656

Pais, R., Lohs, C., Wu, Y., Wang, J., and Aksoy, S. (2008). The obligate mutualist Wigglesworthia glossinidia influences reproduction, digestion, and immunity processes of its host, the tsetse fly. Appl. Environ. Microbiol. 74, 5965-5974.

Pan, X., Zhou, G., Wu, J., Bian, G., Lu, P., Raikhel, A. S., et al. (2012) Wolbachia induces reactive oxygen species (ROS)-dependent activation of the Toll pathway to control dengue virus in the mosquito Aedes aegypti. Proc. Natl. Acad. Sci. U.S.A. 109, E23-E31.

Panteleev, D., Goriacheva, I. I., Andrianov, B. V., Reznik, N. L., Lazebnyi, O. E., and Kulikov, A. M. (2007). The endosymbiotic bacterium Wolbachia enhances the nonspecific resistance to insect pathogens and alters behaviour of Drosophila melanogaster. Genetika 43, 1277-1280.

Pinto, S. B., Mariconti, M., Bazzocchi, C., Bandi, C., and Sinkins, S. P. (2012). Wolbachia surface protein induces innate immune responses in mosquito cells. BMC Microbiol. 12:S11. doi: 10.1186/1471-2180-12S1-S11

Rancès, E., Ye, Y. H., Woolfit, M., McGraw, E. A., and O'Neill, S. L. (2012). The relative importance of innate immune priming in Wolbachia-mediated dengue interference. PLoS Pathog. 8:e1002548. doi: 10.1371/journal.ppat.1002548

Regassa, L. B., and Gasparich, G. E. (2006). Spiroplasmas: evolutionary relationships and biodiversity. Front. Biosci. 11, 2983-3002. doi: $10.2741 / 2027$
Rottschaefer, S. M., and Lazzaro, B. P. (2012). No effect of Wolbachia on resistance to intracellular infection by pathogenic bacteria in Drosophila melanogaster. PLoS ONE 7:e40500. doi: 10.1371/journal.pone.0040500

Royet, J. (2011). Epithelial homeostasis and the underlying molecular mechanisms in the gut of the insect model Drosophila melanogaster. Cell. Mol. Life Sci. 68, 3651-3660.

Ryu, J. H., Ha, E. M., and Lee, W. J. (2010). Innate immunity and gutmicrobe mutualism in Drosophila. Dev. Comp. Immunol. 34, 369-376.

Sadd, B. M., and Schmid-Hempel, P. (2009). "Ecological and evolutionary implications of specific immune responses," in Insect Infection and Immunity, eds J. Rolff and S. E. Reynolds (Oxford: Oxford University Press), 225-240.

Saridaki, A., and Bourtzis, K. (2010). Wolbachia: more than just a bug in insects genitals. Curr. Opin. Microbiol. 13, 67-72.

Schmitz, A., Anselme, C., Ravallec, M., Rebuf, C., Simon, J. C., Gatti, J. L., et al. (2012). The cellular immune response of the pea aphid to foreign intrusion and symbiotic challenge. PLOS ONE 7:e42114. doi: 10.1371/journal.pone.0042114

Schneider, D. (2009). "Physiological integration of innate immunity," in Insect Infection and Immunity, eds J. Rolff and S. E. Reynolds (Oxford: Oxford University Press), 106-116.

Shaw, A. E., Veronesi, E., Maurin, G., Ftaich, N., Guiqen, F., Rixon, F., et al. (2012). Drosophila melanogaster as a model organism for bluetongue virus replication and tropism. J. Virol. 86, 9015-9024.

Teixeira, L., Ferreira, A., and Ashburner, M. (2008). The bacterial symbiont Wolbachia induces resistance to RNA viral infections in Drosophila melanogaster. PLoS Biol. 6:e2. doi: 10.1371/journal.pbio.1000002

Thomas, P., Kenny, N., Eyles, D., Moreira, L. A., O'Neill, S. L., and Asgari, S. (2011). Infection with the $w \mathrm{Mel}$ and $w$ MelPop strains of Wolbachia leads to higher levels of melanization in the hemolymph of Drosophila melanogaster, Drosophila simulans and Aedes aegypti. Dev. Comp. Immunol. 35, 360-365.

Turley, A. P., Moreira, L. A., O'Neill, S. L., and McGraw, E. A. (2009). Wolbachia infection reduces bloodfeeding success in the dengue fever mosquito, Aedes aegypti. PLoS Negl. Trop. Dis. 3:e516. doi: 10.1371/ journal.pntd.0000516

Vigneron, A., Charif, D., VincentMonégat, C., Vallier, A., Gavory, 
F., Wincker, P., et al. (2012). Host gene response to endosymbiont and pathogen in the cereal weevil Sitophilus oryzae. BMC Microbiol. 12:S14. doi: 10.1186/1471-2180-12S1-S14

Walker, T., Johnson, P. H., Moreira, L. A., Iturbe-Ormaetxe, I., Frentiu, F. D., McMeniman, C. J., et al. (2011). The wMel Wolbachia strain blocks dengue and invades caged Aedes aegypti populations. Nature 476, 450-453.

Wang, J., and Aksoy, S. (2012). PGRP-LB is a maternally transmitted immune milk protein that influences symbiosis and parasitism in tsetse's offspring. Proc. Natl. Acad. Sci. U.S.A. 109, 10552-10557.

Wang, J., Wu, Y., Yang, G., and Aksoy, S. (2009). Interactions between mutualist Wigglesworthia and tsetse peptidoglycan recognition protein (PGRP-LB) influence trypanosome transmission. Proc. Natl. Acad. Sci. U.S.A. 106, 12133-12138.

Weiss, B. L., Maltz, M., and Aksoy, S. (2012). Obligate symbionts activate immune system development in the tsetse fly. J. Immunol. 188, 3395-3403.
Weiss, B. L., Wu, Y., Schwank, J. J., Tolwinski, N. S., and Aksoy, S. (2008). An insect symbiosis is influenced by bacterium-specific polymorphisms in outer-membrane protein A. Proc. Natl. Acad. Sci. U.S.A. 105, 15088-15093.

Weiss, B., and Aksoy, S. (2011). Microbiome influences on insect host vector competence. Trends Parasitol. 27, 514-522.

Wernegreen, J. J. (2012). Strategies of genomic integration within insectbacterial mutualisms. Biol. Bull. 223, 112-122.

Werren, J. H., Baldo, L., and Clark, M. E. (2008). Wolbachia: master manipulators of invertebrate biology. Nat. Rev. Microbiol. 6, 741-751.

Wong, Z. S., Hedges, L. M., Brownlie, J. C., and Johnson, K. N. (2011). Wolbachia-mediated antibacterial protection and immune gene regulation in Drosophila. PLoS ONE 6:e25430. doi: 10.1371/journal.pone.0025430

Xi, Z., Gavotte, L., Xie, Y., and Dobson, S. (2008). Genome-wide analysis of the interaction between the endosymbiotic bacterium Wolbachia and its Drosophila host. BMC Genomics 9:1. doi: 10.1186/1471-2164-9-1
Xie, J., Tiner, B., Vilchez, I., and Mateos, M. (2011). Effect of the Drosophila endosymbiont Spiroplasma on parasitoid wasp development and on the reproductive fitness of waspattacked fly survivors. Evol. Ecol. 25, 1065-1079.

Xie, J., Vilchez, I., and Mateos, M. (2010). Spiroplasma bacteria enhance survival of Drosophila hydei attacked by the parasitic wasp Leptopilina heterotoma. PLoS ONE 5:e12149. doi: 10.1371/journal.pone.0012149

Zélé, F., Nicot, A., Duron, A., and Rivero, A. (2012). Infection with Wolbachia protects mosquitoes against Plasmodium-induced mortality in a natural system. J. Evol. Biol. 25, 1243-1252.

Zheng, Y., Wang, J. L., Liu, C., Wang, C. P., Walker, T., and Wang, Y. F. (2011). Differentially expressed profiles in the larval testes of Wolbachia infected and uninfected Drosophila. BMC Genomics 12:595. doi: 10.1186/ 1471-2164-12-595

Zouache, K., Michelland, R. J. Failloux, A. B., Grundmann, G. L., and Mavinqui, P. (2012). Chikungunya virus impacts the diversity of symbiotic bacteria in mosquito vector. Mol. Ecol. 21, 2297-2309.

Conflict of Interest Statement: The authors declare that the research was conducted in the absence of any commercial or financial relationships that could be construed as a potential conflict of interest.

Received: 20 December 2012; accepted: 27 February 2013; published online: 15 March 2013.

Citation: Eleftherianos I, Atri J, Accetta $J$ and Castillo JC (2013) Endosymbiotic bacteria in insects: guardians of the immune system? Front. Physiol. 4:46. doi: 10.3389/fphys.2013.00046

This article was submitted to Frontiers in Invertebrate Physiology, a specialty of Frontiers in Physiology.

Copyright (C) 2013 Eleftherianos, Atri, Accetta and Castillo. This is an open-access article distributed under the terms of the Creative Commons Attribution License, which permits use, distribution and reproduction in other forums, provided the original authors and source are credited and subject to any copyright notices concerning any third-party graphics etc. 